\title{
Preoperative Factors Associated with Extrathyroidal Extension in Papillary Thyroid Cancer
}

\author{
Chi-Yu Kuo $^{a}$ Po-Sheng Yang ${ }^{a}$ Ming-Nan Chien ${ }^{b}$ Shih-Ping Cheng ${ }^{a, c}$ \\ ${ }^{a}$ Department of Surgery, MacKay Memorial Hospital and Mackay Medical College, Taipei, Taiwan; \\ ${ }^{b}$ Division of Endocrinology and Metabolism, Department of Internal Medicine, MacKay Memorial Hospital and \\ Mackay Medical College, Taipei, Taiwan; ' Department of Pharmacology, School of Medicine, College of Medicine, \\ Taipei Medical University, Taipei, Taiwan
}

\section{Keywords}

Extrathyroidal extension $\cdot$ Age $\cdot$ Body mass index $\cdot$ BRAF .

Thyroid carcinoma

\begin{abstract}
Objective: Extrathyroidal extension may not be accurately recognized during thyroidectomy and can increase the risk of positive margins and even recurrence. This study aimed to investigate the preoperative factors associated with extrathyroidal extension. Methods: We analyzed 887 patients with papillary thyroid cancer (PTC) who underwent surgery in the period of 2005-2017. Binary logistic regression analyses and generalized additive models were used to identify associations. Results: Minimal extrathyroidal extension was present in $233(26 \%)$ patients and advanced extrathyroidal extension was found in 60 (7\%) patients. Age, BMI, and tumor size were independent predictors of all or advanced extrathyroidal extension. Among the 493 patients whose BRAF mutation status was available, age $(\mathrm{OR}=1.025), \mathrm{BMI}(\mathrm{OR}=$ $1.091)$, tumor size $(\mathrm{OR}=1.544)$, and BRAF V600E mutation $(O R=2.311)$ were independently associated with extrathyroidal extension. Conclusions: Older age, a greater BMI, a larger tumor size, and presence of the BRAF mutation were
\end{abstract}

predictive of extrathyroidal extension. These factors should be taken into consideration in decision-making before surgery is performed.

(c) 2020 European Thyroid Association Published by S. Karger AG, Basel

\section{Introduction}

Papillary thyroid cancer (PTC) is the most common endocrine malignancy, and its incidence is rising worldwide. Although PTC generally follows an indolent course, an increase in the mortality rate for advanced-stage PTC has been observed [1]. Standard treatment consists of surgery with or without radioactive iodine (RAI) therapy. For patients who have RAI-refractory thyroid cancer, the management options are limited [2]. A few clinicopathologic factors have been identified to be associated with overall survival (OS) in PTC patients [3]. Gender, age, tumor size, extrathyroidal extension, lymph node metastasis, and distant spread are well-known prognostic factors.

Extrathyroidal extension, by definition, is characterized by tumor extension beyond the thyroid capsule into the adjacent soft tissue. It can be subdivided into 2 types,

Published by S. Karger AG, Basel
Shih-Ping Cheng, $\mathrm{MD}, \mathrm{PhD}$

Department of Surgery, MacKay Memorial Hospital 92 Chung-Shan North Rd., Section 2

Taipei 10449 (Taiwan)

surg.mmh@gmail.com 
i.e., minimal or advanced (extensive). When present, minimal extrathyroidal extension increases the stage of any tumor of $<4 \mathrm{~cm}$ to the T3 category (according to the 7 th edition of the American Joint Committee on Cancer Tumor-Node-Metastasis staging system [AJCC TNM]), and advanced extrathyroidal extension increases it to the T4 category. Nonetheless, the prognostic role of extrathyroidal extension in PTC remains controversial. In the current American Thyroid Association (ATA) guidelines, minimal extrathyroidal extension is considered to indicate an intermediate risk of disease recurrence and/ or persistence [4]. On the contrary, in the 8th edition of the AJCC TNM, T3b is defined as a gross extrathyroidal extension invading strap muscles, and minor extension through the thyroid capsule is not included in the staging.

Proper identification of the presence of extrathyroidal extension has some clinical implications. Studies have shown that patients who have extrathyroidal extension are more likely to have positive margins $[5,6]$. Accordingly, a more meticulous resection may be required to ensure the completeness of disease eradication. In this study, we aimed to investigate the potential preoperative factors associated with extrathyroidal extension in PTC.

\section{Materials and Methods}

\section{Patients}

After obtaining institutional review board approval, we queried the prospectively maintained patient registry for all patients undergoing thyroid surgery [7]. We identified patients diagnosed with PTC who underwent an initial surgical treatment with curative intent at our hospital in the period of 2005-2017. Those who were reclassified as having a noninvasive follicular thyroid neoplasm with papillary-like nuclear features (NIFTP) were not included. Patients younger than 20 years were excluded from this study.

Demographic data including age and gender, as well as body weight and height, were recorded at diagnosis. In Taiwan, overweight and obesity are defined as a BMI $\geq 24$ and $\geq 27$, respectively, because Asians have a higher rate of comorbidity and fat mass at lower BMI levels than Caucasians do [8].

\section{Preoperative Workup}

A preoperative neck ultrasound was performed by endocrinologists and/or endocrine surgeons $[9,10]$. For each thyroid nodule, tumor diameters were measured on the anteroposterior, transverse, and longitudinal planes. Tumor size was defined as the greatest diameter of each lesion. The diagnosis of Hashimoto's thyroiditis was made based on the characteristic ultrasound pattern of the thyroid parenchyma in conjunction with positive serum autoantibodies against thyroid peroxidase and/or thyroglobulin. The
$B R A F$ V600E mutation status was determined by Sanger sequencing on cytological cell blocks or surgical specimens $[11,12]$. The $B R A F$ mutation was for tested selectively before 2015 and routinely after 2015 .

\section{Extrathyroidal Extension}

During the study period, a total thyroidectomy was performed for differentiated thyroid cancer $>1 \mathrm{~cm}$, and lobectomy was done for microcarcinoma [13]. Nonetheless, the operative procedure was finally determined after sharing the decision-making with the patient. All microscopic slides and pathology reports were reviewed by 2 pathologists [14].

The 7th edition of the AJCC TNM criteria was implemented at our institute until the end of 2017. Accordingly, minimal extrathyroidal extension (T3) is characterized by involvement of the sternothyroid muscle or perithyroidal soft tissues and it is generally identified by light microscopic examination. Advanced extrathyroidal extension (T4) is defined as direct extension of the tumor into the subcutaneous soft tissues, larynx, trachea, esophagus, or recurrent laryngeal nerve. No patient in our series had very advanced disease, in which the cancer invades the prevertebral fascia or encases the carotid artery or mediastinal vessels.

\section{Statistical Analysis}

Continuous variables were tested for normality using the D'Agostino-Pearson omnibus K2 normality test. As transformations did not improve normality, nonparametric tests were used throughout. Data are expressed as medians (IQR) or numbers (\%). We treated extrathyroidal extension as an ordered categorical variable. The Cochran-Armitage trend test was used to examine associations with dichotomous parameters, and the Jonckheere-Terpstra test was used for continuous variables [15]. To explore possible associations with BMI, we used the Mann-Whitney U test for dichotomous parameters and the Spearman rank correlation coefficient for continuous variables.

Binary logistic regression analysis was performed to identify preoperative determinants of extrathyroidal extension [16]. Predictors with $p<0.05$ in the univariate logistic regression analysis were included in multivariate models. OR and 95\% CI are reported. We evaluated the performance of the models by calculating the area under the receiver operating characteristic (ROC) curve and the goodness of fit by means of the Hosmer-Lemeshow test. To relax the linearity assumptions, generalized additive models were also plotted and adjusted for appropriate covariates [17].

The secondary analysis incorporated the BRAF mutation status. For visual assessment of the association between BMI and the $B R A F$ mutation, the relationships were fitted using a nonparametric regression method, i.e., locally weighted scatterplot smoothing (LOWESS). The LOWESS regression method does not require any a priori known relationship between the predictor and the dependent variables [18]. Furthermore, we used multiple imputation to account for missing information in cases with an unknown $B R A F$ mutation status. To redeem the bias of different $B R A F$ test policies, a missing $B R A F$ mutation status was imputed using the year of diagnosis. The final imputed dataset was assessed as a sensitivity analysis.

All analyses were performed with Stata v14.0 software (StataCorp., College Station, TX, USA). A two-sided $p<0.05$ was considered statistically significant. 
Table 1. Associations between preoperative parameters and extrathyroidal extension in patients with PTC

\begin{tabular}{|c|c|c|c|c|}
\hline \multirow[t]{2}{*}{ Variable } & \multicolumn{3}{|c|}{ Extrathyroidal extension } & \multirow[t]{2}{*}{$p$ value } \\
\hline & $\begin{array}{l}\text { none } \\
(n=594)\end{array}$ & $\begin{array}{l}\text { minimal } \\
(n=233)\end{array}$ & $\begin{array}{l}\text { advanced } \\
(n=60)\end{array}$ & \\
\hline Age & $45(36-54)$ & $48(39-57)$ & $55(45-62)$ & $<0.001$ \\
\hline Female sex & $497(84)$ & $184(79)$ & $47(78)$ & 0.094 \\
\hline BMI & $23.2(21.1-25.6)$ & $23.8(21.9-27.8)$ & $25.4(22.7-28.4)$ & $<0.001$ \\
\hline Category & & & & $<0.001$ \\
\hline Underweight & $25(4)$ & $8(3)$ & $1(2)$ & \\
\hline Normal & $325(55)$ & $112(48)$ & $18(30)$ & \\
\hline Overweight & $144(24)$ & $47(20)$ & $19(32)$ & \\
\hline Obese & $100(17)$ & $66(28)$ & $22(37)$ & \\
\hline Tumor size & $1.2(0.7-2.0)$ & $1.9(1.3-2.5)$ & $2.9(2.2-3.5)$ & $<0.001$ \\
\hline Hashimoto's thyroiditis & $91(15)$ & $45(19)$ & $4(7)$ & 0.683 \\
\hline BRAF mutation $^{\mathrm{a}}$ & $196(67)$ & $130(81)$ & $38(93)$ & $<0.001$ \\
\hline
\end{tabular}

Values are presented as medians (IQR) or numbers (\%).The total number is patients is 887 . $^{\text {a }}$ Status was available for 493 patients.

Table 2. Univariate and multivariate logistic regression analysis of factors associated with extrathyroidal extension in patients with PTC

\begin{tabular}{|c|c|c|c|c|c|c|c|c|c|c|c|c|}
\hline \multirow[t]{3}{*}{ Variable } & \multicolumn{6}{|c|}{ Extrathyroidal extension (all) } & \multicolumn{6}{|c|}{ Advanced extrathyroidal extension (T4 stage) } \\
\hline & \multicolumn{3}{|c|}{ univariate } & \multicolumn{3}{|c|}{ multivariate } & \multicolumn{3}{|c|}{ univariate } & \multicolumn{3}{|c|}{ multivariate } \\
\hline & OR & $95 \% \mathrm{CI}$ & $p$ value & OR & $95 \% \mathrm{CI}$ & $p$ value & OR & $95 \% \mathrm{CI}$ & $p$ value & OR & $95 \% \mathrm{CI}$ & $p$ value \\
\hline Age & 1.025 & $1.014-1.037$ & $<0.001$ & 1.031 & $1.019-1.044$ & $<0.001$ & 1.042 & $1.022-1.063$ & $<0.001$ & 1.046 & $1.025-1.067$ & $<0.001$ \\
\hline Sex & 0.727 & $0.510-1.037$ & 0.078 & & & & 0.775 & $0.409-1.469$ & 0.435 & & & \\
\hline BMI & 1.094 & $1.055-1.135$ & $<0.001$ & 1.074 & $1.032-1.117$ & $<0.001$ & 1.114 & $1.050-1.183$ & $<0.001$ & 1.081 & $1.010-1.156$ & 0.024 \\
\hline Tumor size & 1.758 & $1.545-2.001$ & $<0.001$ & 1.847 & $1.614-2.114$ & $<0.001$ & 1.915 & $1.588-2.309$ & $<0.001$ & 2.052 & $1.668-2.523$ & $<0.001$ \\
\hline Hashimoto's thyroiditis & 1.110 & $0.760-1.622$ & 0.590 & & & & 0.363 & $0.129-1.017$ & 0.054 & & & \\
\hline
\end{tabular}

\section{Results}

A total of 887 patients with PTC met the inclusion criteria. About $80 \%(n=728)$ were women. The median age at diagnosis was 47 years (range 20-91). The median BMI was 23.6 (range 16.4-43.0), with 34 patients (4\%) being underweight, 455 (51\%) being within the normal range, 210 (24\%) being overweight, and 188 (21\%) being obese. Of these patients, 703 (79\%) underwent a total thyroidectomy and $184(21 \%)$ had a lobectomy. The median tumor size was $1.5 \mathrm{~cm}$, and 262 tumors (30\%) were microcarcinomas. Concurrent Hashimoto's thyroiditis was present in $140(16 \%)$ patients.

Based on clinical features and pathologic examination, $594(67 \%)$ patients had no extrathyroidal extension, 233 (26\%) presented with minimal extrathyroidal extension, and $60(7 \%)$ had advanced extrathyroidal extension. As shown in Table 1, extrathyroidal extension in PTC was associated with an older age, a greater BMI, and a larger tu- mor size. Interestingly, the prevalence of overweight and obesity was 41,48 , and $68 \%$ in patients with no, minimal, and advanced extrathyroidal extension, respectively. BMI was positively associated with age (Spearman's $\rho=0.201$, $p<0.001$ ), and men had significantly higher BMI than women (median 24.6 vs. $23.2, p<0.001$ ). BMI was not associated with tumor size or Hashimoto's thyroiditis.

Next, we employed logistic regression to model the probability of extrathyroidal extension based on these preoperative factors. Consistently, age, BMI, and tumor size were independent predictors of extrathyroidal extension in PTC (Table 2). The area under the ROC curve was 0.741 (95\% CI 0.707-0.775) with a good fit (HosmerLemeshow test, $p=0.240$ ). Additionally, OR were estimated using generalized additive models. Of interest, the OR for extrathyroidal extension increased in a nearly linear fashion along with increasing patient age or BMI (Fig. 1). An inverted U-shaped relationship was found for tumor size after adjustment for age, sex, and BMI. 


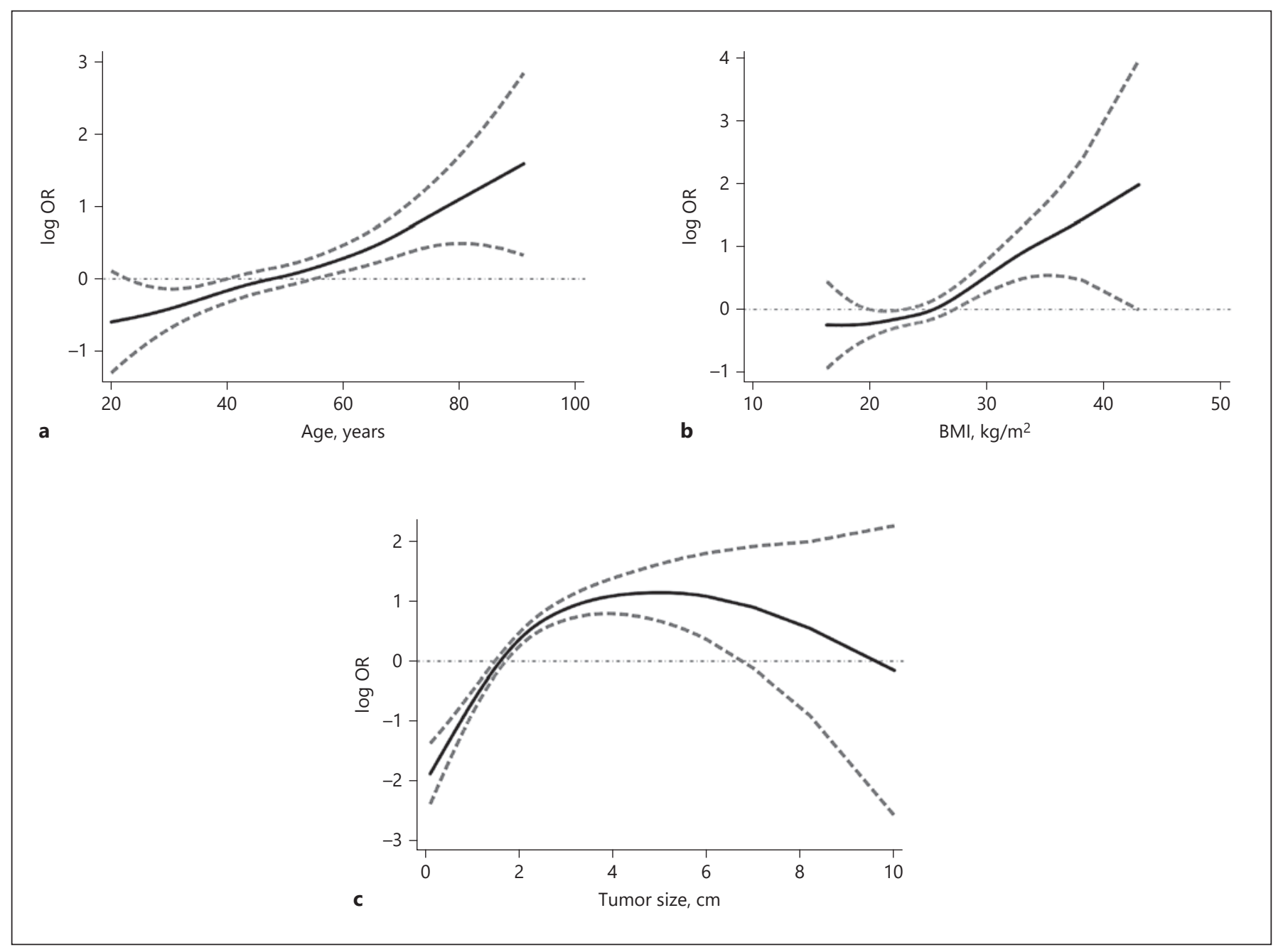

Fig. 1. Log-transformed OR and 95\% CI (dashed lines) for extrathyroidal extension at a given age (a), BMI (b), or tumor diameter (c) in 887 patients with PTC. Estimates were obtained from generalized additive models adjusted for sex (a), sex and age (b), and sex, age, and BMI (c), respectively.

We further analyzed the potential factors associated with advanced extrathyroidal extension. In agreement with a previous analysis, age $(\mathrm{OR}=1.046), \mathrm{BMI}(\mathrm{OR}=$ $1.081)$, and tumor size $(\mathrm{OR}=2.052)$ were independently associated with advanced extrathyroidal extension (Table 2 ). The area under the ROC curve was 0.835 (95\% CI $0.787-0.884$ ). The model was well fitted (Hosmer-Lemeshow test, $p=0.483$ ).

$B R A F$ mutation status data were available for 493 patients (56\%); 364 (74\%) carried the BRAF V600E mutation. The presence of the BRAF mutation was associated with an older age $(p=0.002)$ and a higher BMI $(p=0.015)$ but not with gender or tumor size. We further explored the association between $\mathrm{BMI}$ and the BRAF mutation by modeling the relationship using a LOWESS plot (Fig. 2).
The plot suggested that increasing BMI did indeed correlate with a higher prevalence of the $B R A F$ mutation in PTC.

Given that it is possible to obtain the BRAF mutation status by fine-needle aspiration biopsy of thyroid nodules, we performed a secondary analysis that incorporated the BRAF mutation status. As shown in Table 3, age $(\mathrm{OR}=1.025)$, $\mathrm{BMI}(\mathrm{OR}=1.091)$, tumor size $(\mathrm{OR}=1.544)$, and $B R A F$ mutation $(\mathrm{OR}=2.311)$ were independent predictors of extrathyroidal extension in PTC. The area under the ROC curve was 0.714 (95\% CI 0.667-0.760). The model overall fits the data well (Hosmer-Lemeshow test, $p=0.313)$.

The BRAF mutation was tested for selectively before 2015. To account for the possibility that only the more 
Table 3. Univariate and multivariate logistic regression analysis of factors (incorporating the status of BRAF mutation) associated with extrathyroidal extension in patients with PTC

\begin{tabular}{|c|c|c|c|c|c|c|}
\hline \multirow[t]{2}{*}{ Variable } & \multicolumn{3}{|c|}{ Univariate } & \multicolumn{3}{|c|}{ Multivariate } \\
\hline & OR & $95 \% \mathrm{CI}$ & $p$ value & OR & $95 \% \mathrm{CI}$ & $p$ value \\
\hline Age & 1.026 & $1.012-1.040$ & $<0.001$ & 1.025 & $1.009-1.040$ & 0.002 \\
\hline Sex & 0.792 & $0.498-1.260$ & 0.325 & & & \\
\hline Body mass index & 1.115 & $1.064-1.169$ & $<0.001$ & 1.091 & $1.038-1.147$ & 0.001 \\
\hline Tumor size & 1.457 & $1.236-1.717$ & $<0.001$ & 1.544 & $1.291-1.846$ & $<0.001$ \\
\hline Hashimoto's thyroiditis & 0.788 & $0.502-1.237$ & 0.300 & & & \\
\hline BRAF mutation & 2.494 & $1.596-3.895$ & $<0.001$ & 2.311 & $1.438-3.713$ & 0.001 \\
\hline
\end{tabular}

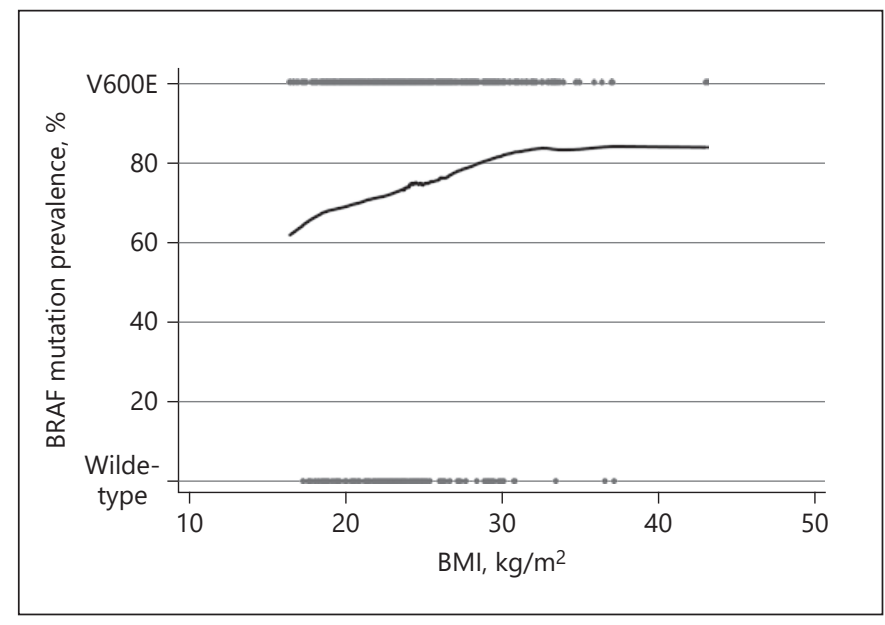

Fig. 2. Plot depicting the relationship between BMI and the status of BRAF mutation in 493 patients with PTC. The trend is shown by the LOWESS line. Degree of smoothing $=0.9$.

aggressive tumors were tested for, iterative rounds of imputation $(n=20)$ were performed to impute the BRAF mutation status based on the year of diagnosis. The results from the sensitivity analysis using multiple imputation showed no remarkable difference (Table 4). This indicates that, where data were missing, this did not cause any considerable bias in the estimation of regression coefficients.

\section{Discussion}

It is generally accepted that advanced extrathyroidal extension negatively impacts outcomes in PTC. The 2015 ATA guidelines classified macroscopic invasion into the perithyroidal soft tissues as high-risk disease and recommended a near-total or total thyroidectomy with RAI adjuvant therapy in patients with gross extrathyroidal ex-
Table 4. Multivariate logistic regression analysis of factors associated with extrathyroidal extension after multiple imputation of missing data on the status of BRAF mutation in patients with PTC

\begin{tabular}{lllr}
\hline Variable & OR & $95 \%$ CI & $p$ value \\
\hline Age & 1.030 & $1.017-1.043$ & $<0.001$ \\
BMI & 1.070 & $1.029-1.114$ & 0.001 \\
Tumor size & 1.862 & $1.623-2.136$ & $<0.001$ \\
BRAF mutation & 1.820 & $1.144-2.896$ & 0.012 \\
\hline
\end{tabular}

tension [4]. Likewise, RAI adjuvant therapy should be considered after a total thyroidectomy in intermediaterisk patients who have minimal extrathyroidal extension. Despite controversy, tumors with minimal extrathyroidal extension might have a higher recurrence rate than those with no extrathyroidal extension [19-21]. An analysis of the National Cancer Database demonstrated that even a minimal extrathyroidal extension was associated with a compromised overall survival [22]. Interestingly, our findings suggest that minimal and advanced extrathyroidal extension have similar associations with some of the preoperative factors.

Although extrathyroidal extension bears some prognostic significance, intraoperative inspection for extrathyroidal extension is often inaccurate [23]. Intraoperative frozen sections may be helpful to identify the presence of extrathyroidal extension [24], but the number of frozen sections has dramatically decreased in recent years. Preoperative neck ultrasound plays a pivotal role in characterization and staging of thyroid nodules. Common sonographic features of extrathyroidal extension include a thyroid capsular distortion or abutment, contour bulging, a long interface between the tumor and the thyroid capsule, and a tracheal footprint. It is not difficult to discern advanced extrathyroidal extension on ultrasound. Nonetheless, the positive predictive value of predicting 
minimal extrathyroidal extension is modest $[25,26]$. In this context, proper identification of associated factors may alert physicians to the presence of extrathyroidal extension.

We found that $B R A F$ mutation, tumor size, BMI, and age were independent predictive factors. Of these factors, $B R A F$ mutation is the most common oncogenic driver of PTC but there are wide global variations in the occurrence rate. $B R A F$ mutation analysis has a high rule-in capacity in cytologically indeterminate thyroid nodules. Additionally, previous studies have established a positive association between the BRAF V600E mutation and most of the clinical features of aggressiveness, including extrathyroidal extension, lymph node metastasis, and an advanced stage $[11,27]$. Nonetheless, this information is not always available before surgery.

Consistent with a previous report [28], we found that tumor size was an independent risk factor for extrathyroidal extension. This is comprehensible because continuous tumor growth would lead to a higher likelihood of extension beyond the thyroid capsule, particularly in those tumors in the periphery. An older age was also independently associated with extrathyroidal extension. We previously reported that an older age was associated with a glycolytic phenotype of PTC [29]. Local acidification of the tumor microenvironment in association with the Warburg effect may potentially support tumor invasion [30]. Furthermore, aging restrains adaptive immunity and renders the tumor microenvironment more immunosuppressive, which may facilitate the invasion process [31].

An intriguing finding in this study is that a higher BMI of patients was modestly but independently associated with extrathyroidal extension. Overweight and obesity are associated with a higher risk of the occurrence of thyroid cancer [32]. Additionally, obese patients with PTC have an increased risk of developing locoregional events during follow-up [33]. In a large Korean series, higher BMI could predict multiplicity and extrathyroidal extension, whereas a higher body surface area was a predictor for multiplicity [34]. Possible mechanisms involving the link between adiposity and cancer include insulin resistance, adipokine, and subclinical inflammation. We have demonstrated that leptin and adiponectin receptors are present in PTC and have correlations with some clinicopathologic features [35-37]. Recently, Pitoia et al. [38] found that the state of insulin resistance was associated with structural persistent disease. It is noteworthy that we observed a positive association between BMI and BRAF V600E mutation. These findings are in line with an early study [39]. Further research is necessary to elucidate the mechanisms underlying the link between obesity and BRAF mutation.

In conclusion, age, BMI, tumor size, and BRAF mutation are preoperative factors associated with extrathyroidal extension in PTC. Although BMI is typically not considered a prognostic variable, it is the only modifiable risk factor. It would be of great interest to know whether weight reduction is helpful in terms of long-term outcomes in obese patients with PTC.

\section{Statement of Ethics}

This study was approved by the Institutional Review Board of the MacKay Memorial Hospital. All of the procedures involving human participants were in accordance with the ethics standards of the MacKay Memorial Hospital and the 1964 Helsinki Declaration and its later amendments or comparable standards. For this type of study, formal consent is waived.

\section{Disclosure Statement}

The authors have no conflict of interests to declare.

\section{Funding Sources}

No funding was received.

\section{Author Contributions}

Study conception and design: C.-Y.K., P.-S.Y., and S.-P.C. Acquisition of data: C.-Y.K. and S.-P.C. Analysis and interpretation of data: C.-Y.K., P.-S.Y., M.-N.C., and S.-P.C. Drafting of this paper: C.-Y.K. and S.-P.C. Critical revision: P.-S.Y. and M.-N.C. All of the authors read and approved the final version of this paper.
References
1 Lim H, Devesa SS, Sosa JA, Check D, Kitahara $\mathrm{CM}$. Trends in thyroid cancer incidence and mortality in the United States, 1974-2013. JAMA. 2017 Apr;317(13):1338-48.
2 Fugazzola L, Elisei R, Fuhrer D, Jarzab B, Le- boulleux S, Newbold K, et al. 2019 European Thyroid Association Guidelines for the Treat- ment and Follow-Up of Advanced Radioio- dine-Refractory Thyroid Cancer. Eur Thy- roid J. 2019 Oct;8(5):227-45.
3 Ito Y, Miyauchi A, Kihara M, Fukushima M, Higashiyama T, Miya A. Overall survival of papillary thyroid carcinoma patients: a single- institution long-term follow-up of $5897 \mathrm{pa}-$ tients. World J Surg. 2018 Mar;42(3):615-22. 
4 Haugen BR, Alexander EK, Bible KC, Doherty GM, Mandel SJ, Nikiforov YE, et al. 2015 American Thyroid Association Management Guidelines for Adult Patients with Thyroid Nodules and Differentiated Thyroid Cancer: The American Thyroid Association Guidelines Task Force on Thyroid Nodules and Differentiated Thyroid Cancer. Thyroid. 2016 Jan;26(1):1-133.

5 Wang LY, Ghossein R, Palmer FL, Nixon IJ, Tuttle RM, Shaha AR, et al. Microscopic positive margins in differentiated thyroid cancer is not an independent predictor of local failure. Thyroid. 2015 Sep;25(9):993-8.

6 Abraham E, Tran B, Roshan D, Graham S, Lehane $\mathrm{C}$, Wykes J, et al. Microscopic positive margins in papillary thyroid cancer do not impact disease recurrence. ANZ J Surg. 2018 Nov;88(11):1193-7.

7 Cheng SP, Liu CL, Tzen CY, Yang TL, Jeng KS, Liu TP, et al. Characteristics of well-differentiated thyroid cancer associated with multinodular goiter. Langenbecks Arch Surg. 2008 Sep;393(5):729-32.

8 Huang KC. Obesity and its related diseases in Taiwan. Obes Rev. 2008 Mar;9(s1 Suppl 1): 32-4.

9 Cheng SP, Lee JJ, Liu TP, Lee KS, Liu CL. Preoperative ultrasonography assessment of vocal cord movement during thyroid and parathyroid surgery. World J Surg. 2012 Oct; 36(10):2509-15.

10 Cheng SP, Lee JJ, Lin JL, Chuang SM, Chien MN, Liu CL. Characterization of thyroid nodules using the proposed thyroid imaging reporting and data system (TI-RADS). Head Neck. 2013 Apr;35(4):541-7.

11 Cheng SP, Hsu YC, Liu CL, Liu TP, Chien MN, Wang TY, et al. Significance of allelic percentage of BRAF c.1799T > A (V600E) mutation in papillary thyroid carcinoma. Ann Surg Oncol. 2014 Dec;21(S4 Suppl 4):S61926.

12 Wang TY, Liu CL, Chen MJ, Lee JJ, Pun PC, Cheng SP. Expression of haem oxygenase-1 correlates with tumour aggressiveness and BRAF V600E expression in thyroid cancer. Histopathology. 2015 Feb;66(3):447-56.

13 Lee F, Yang PS, Chien MN, Lee JJ, Leung CH, Cheng SP. An increased neutrophil-to-lymphocyte ratio predicts incomplete response to therapy in differentiated thyroid cancer. Int J Med Sci. 2018 Nov;15(14):1757-63.

14 Cheng SP, Chien MN, Wang TY, Lee JJ, Lee CC, Liu CL. Reconsideration of tumor size threshold for total thyroidectomy in differentiated thyroid cancer. Surgery. 2018 Sep; 164(3):504-10.

15 Aihara H, Tago M, Oishi T, Katsuki NE, Yamashita SI. Visual impairment, partially dependent ADL and extremely old age could be predictors for severe fall injuries in acute care settings. Int J Gerontol. 2018;12(3):175-9.
16 Lee JJ, Wang TY, Liu CL, Chien MN, Chen MJ, Hsu YC, et al. Dipeptidyl peptidase IV as a prognostic marker and therapeutic target in papillary thyroid carcinoma. J Clin Endocrinol Metab. 2017 Aug;102(8):2930-40.

17 Hastie T, Tibshirani R. Generalized additive models for medical research. Stat Methods Med Res. 1995 Sep;4(3):187-96.

18 Cleveland WS, Devlin SJ. Locally weighted regression: an approach to regression analysis by local fitting. J Am Stat Assoc. 1988;83(403): 596-610.

19 Park JS, Chang JW, Liu L, Jung SN, Koo BS. Clinical implications of microscopic extrathyroidal extension in patients with papillary thyroid carcinoma. Oral Oncol. 2017 Sep;72: 183-7.

20 Amit M, Boonsripitayanon M, Goepfert RP, Tam S, Busaidy NL, Cabanillas ME, et al. Extrathyroidal extension: does strap muscle invasion alone influence recurrence and survival in patients with differentiated thyroid cancer? Ann Surg Oncol. 2018 Oct;25(11): $3380-8$.

21 Diker-Cohen T, Hirsch D, Shimon I, Bachar G, Akirov A, Duskin-Bitan H, et al. Impact of minimal extrathyroid extension in differentiated thyroid cancer: systematic review and meta-analysis. J Clin Endocrinol Metab. 2018 Mar;103(6):2100-6.

22 Youngwirth LM, Adam MA, Scheri RP, Roman SA, Sosa JA. Extrathyroidal extension is associated with compromised survival in patients with thyroid cancer. Thyroid. 2017 May;27(5):626-31.

23 Jung SP, Kim M, Choe JH, Kim JS, Nam SJ, Kim JH. Clinical implication of cancer adhesion in papillary thyroid carcinoma: clinicopathologic characteristics and prognosis analyzed with degree of extrathyroidal extension. World J Surg. 2013 Jul;37(7):1606-13.

24 Park YM, Wang SG, Goh JY, Shin DH, Kim IJ, Lee BJ. Intraoperative frozen section for the evaluation of extrathyroidal extension in papillary thyroid cancer. World J Surg. 2015 Jan;39(1):187-93.

25 Kamaya A, Tahvildari AM, Patel BN, Willmann JK, Jeffrey RB, Desser TS. Sonographic detection of extracapsular extension in papillary thyroid cancer. J Ultrasound Med. 2015 Dec;34(12):2225-30.

26 Kuo EJ, Thi WJ, Zheng F, Zanocco KA, Livhits MJ, Yeh MW. Individualizing surgery in papillary thyroid carcinoma based on a detailed sonographic assessment of extrathyroidal extension. Thyroid. 2017 Dec;27(12): 1544-9.

27 Gandolfi G, Sancisi V, Piana S, Ciarrocchi A. Time to re-consider the meaning of BRAF V600E mutation in papillary thyroid carcinoma. Int J Cancer. 2015 Sep;137(5):1001-11.
28 Lee HS, Park C, Kim SW, Park T, Chun BK, Hong JC, et al. Correlation of minimal extrathyroidal extension with pathologic features of lymph node metastasis in patients with papillary thyroid carcinoma. J Surg Oncol. 2015 Nov; 112(6):592-6.

29 Hsu YC, Liu CL, Yang PS, Tsai CH, Lee JJ, Cheng SP. Interaction of age at diagnosis with transcriptional profiling in papillary thyroid cancer. World J Surg. 2016 Dec;40(12):29229.

30 Hay N. Reprogramming glucose metabolism in cancer: can it be exploited for cancer therapy? Nat Rev Cancer. 2016 Oct;16(10):63549.

31 Wu Y, Wei J, Chen X, Qin Y, Mao R, Song J, et al. Comprehensive transcriptome profiling in elderly cancer patients reveals aging-altered immune cells and immune checkpoints. Int J Cancer. 2019 Apr;144(7):1657-63.

32 Schmid D, Ricci C, Behrens G, Leitzmann MF. Adiposity and risk of thyroid cancer: a systematic review and meta-analysis. Obes Rev. 2015 Dec;16(12):1042-54.

33 Trésallet C, Seman M, Tissier F, Buffet C, Lupinacci RM, Vuarnesson $\mathrm{H}$, et al. The incidence of papillary thyroid carcinoma and outcomes in operative patients according to their body mass indices. Surgery. 2014 Nov; 156(5): 1145-52.

34 Kim SK, Woo JW, Park I, Lee JH, Choe JH, $\mathrm{Kim} \mathrm{JH}$, et al. Influence of body mass index and body surface area on the behavior of papillary thyroid carcinoma. Thyroid. 2016 May; 26(5):657-66.

35 Cheng SP, Chi CW, Tzen CY, Yang TL, Lee JJ, Liu TP, et al. Clinicopathologic significance of leptin and leptin receptor expressions in papillary thyroid carcinoma. Surgery. 2010 Jun; 147(6):847-53

36 Cheng SP, Yin PH, Hsu YC, Chang YC, Huang SY, Lee JJ, et al. Leptin enhances migration of human papillary thyroid cancer cells through the PI3K/AKT and MEK/ERK signaling pathways. Oncol Rep. 2011 Nov; 26(5):1265-71.

37 Cheng SP, Liu CL, Hsu YC, Chang YC, Huang SY, Lee JJ. Expression and biologic significance of adiponectin receptors in papillary thyroid carcinoma. Cell Biochem Biophys. 2013 Mar;65(2):203-10.

38 Pitoia F, Abelleira E, Bueno F, Urciuoli C, Schmidt A, Niepomniszcze H. Insulin resistance is another factor that increases the risk of recurrence in patients with thyroid cancer. Endocrine. 2015 Apr;48(3):894-901.

39 Lee J, Lee CR, Ku CR, Kang SW, Jeong JJ, Shin DY, et al. Association between obesity and BRAFV600E mutation status in patients with papillary thyroid cancer. Ann Surg Oncol. 2015 Dec;22(S3 Suppl 3):S683-90. 